\title{
Forest remote sensing: inversion using a coherent scattering model and a genetic algorithm
}

\author{
M. Kanj* ${ }^{(1)}$, C. Dahon ${ }^{(2)}$, H. Roussel ${ }^{(2)}$ and B. Duchêne ${ }^{(1)}$
}

(1) Département de Recherche en Electromagnétisme, Laboratoire des Signaux et Systèmes (UMR 8506 : CNRS - Supelec - Univ Paris-Sud 11), Gif-Sur-Yvette, France

(2) L2E, UR2, UPMC univ Paris 6, Paris, France

In remote sensing for forest monitoring, Synthetic Aperture Radar (SAR) imaging is a powerful tool to investigate wide forested areas, particularly at low frequencies (less than $1 \mathrm{GHz}$ ) where the electromagnetic waves penetrate deeply under the forest canopy. Radar measurements allow us to obtain information on the forest biomass, such as density, heights and dielectric properties of trees. In order to extract this information, it is necessary to analyze the wave-tree interactions involved when SAR measurements are performed. At low frequencies, the small branches and leaves of trees do not significantly contribute to the electromagnetic fields scattered by a forest area and can be safely neglected. The main contributions to the scattered field come from the trunks and primary branches. In this context, we have built up an approximate model where the latter are considered as finite circular cylinders. The total scattered field is then obtained by coherently adding the contributions of the different trunks and branches and the contribution of each of them is obtained through the coherent sum of various elementary scattering mechanisms such as single, double and triple bounces.

In the above-mentioned approximate model, the field scattered by a finite circular cylinder is supposed to be radiated by currents flowing on its surface that are the same than those that would flow on an infinite cylinder, except that they are limited to the real length of the cylinder. The model is then completed in order to satisfy the polarimetric reciprocity properties of the double bounce scattering mechanism observed in monostatic configurations for a tilted cylinder. This approximate model is very fast and yields good results as compared to a rigorous model based upon an integral representation of the fields.

The second step is now to use this fast model for inversion purposes. The goal is to evaluate forest parameters such as radius, height, electromagnetic properties or density of trees, from the scattered fields measured in frequency diverse, multipolarization multi-static configurations. This is done by means of a genetic algorithm, through an iterative minimization of a cost functional that expresses the discrepancy between the measured fields and the fields computed by means of the approximate model. The inversion tool is tested on two configurations: the first one involves a single trunk placed over a flat ground and the second one concerns several trees with primary branches. Both inversions are performed from synthetic data obtained by means of the above-mentioned rigorous model. The next step is to test the inversion algorithm on laboratory controlled experimental data collected on a reduced size model forest placed in an anechoic chamber. 